\title{
Design of a Low Phase Noise VCO Used for LTE Band40
}

\author{
Qingyun $\mathrm{Ju}^{1, \mathrm{a}}$, Xinwei $\mathrm{Li}^{1, \mathrm{~b}}$, Liang Tang ${ }^{2, \mathrm{c}}$, Donghai Qiao ${ }^{2, \mathrm{~d}}$
}

\author{
${ }^{1}$ School of Electronic and Information Engineering, Soochow University, Suzhou, 215006, China \\ ${ }^{2}$ Institute of Acoustics, Chinese Academy of Sciences, Beijing, 100190, China \\ aemail: cljqy@126.com, bemail: Ixw91@hotmail.com, cemail: tangliang@mail.ioa.ac.cn, \\ demail: qiaodh@suda.edu.cn
}

Keywords: VCO; LTE Band40; Low Phase Noise; Negative Resistance Analysis Method

\begin{abstract}
According to the frequency of LTE Band40 from 2300MHz to $2400 \mathrm{MHz}$ using for $4 \mathrm{G}$ mobile communication distributed by Ministry of Industry and Information Technology of China, a low phase noise, small size and low power consumption voltage-controlled oscillator (VCO) which can be used in mobile phones supporting 4G LTE mode is designed. The VCO based on a modified Clapp circuit topology is analyzed with the negative resistance analysis method and simulated in the time domain and frequency domain, respectively. Finally, the actual circuit is fabricated and tested. The test results show that the size of the VCO is $11 \mathrm{~mm}$ by $9 \mathrm{~mm}$, the phase noises of the VCO are $-53.79 \mathrm{dBc} / \mathrm{Hz} @ 300 \mathrm{~Hz}$ and $-67.42 \mathrm{dBc} / \mathrm{Hz} @ 1 \mathrm{KHz}$, the voltage-controlled tuning sensitivity is about $24.4 \mathrm{MHz} / \mathrm{V}$ which results in an operating frequency range from $2294 \mathrm{MHz}$ to $2416 \mathrm{MHz}$ up to $5 \mathrm{~V}$ reverse bias voltage of the varactor and the output power is about $1 \mathrm{dBm}$, meeting the application requirements of the $4 \mathrm{G}$ mobile communication well.
\end{abstract}

\section{Introduction}

The data transmission speed of the $4 \mathrm{G}$ mobile communication is much faster than $3 \mathrm{G}$ technology and GSM technology [1 3]. Especially in recent years, the 4G mobile communication technology and products have realized rapid development and more and more districts around the whole world are covered with this kind of high-speed and effective communication networks and systems. Based on the working mechanism of the communication systems of modern mobile phones and similar devices, a mixer module with a local RF VCO is always used for frequency conversion during the process of transmitting or receiving signals. This VCO can generate an RF signal with proper frequency determined by different communication standards of the mobile devices to provide the transmitted or received signals an appropriate high frequency carrier signal. In this paper, a low phase noise VCO whose operating frequency can cover the LTE Band40 [4] is designed with the modified Clapp oscillator, serving as the local RF oscillator in the mobile communication systems. Firstly, a capacitive voltage divider is utilized to push the active device into an unstable state and simultaneously to generate a sufficient negative resistance across the circuit during the frequency range from $2300 \mathrm{MHz}$ to $2400 \mathrm{MHz}$. Then the frequency tuning circuit composed of a varactor, some inductors and capacitors is designed to achieve resonance near $2350 \mathrm{MHz}$ with suitable frequency tuning range implemented by the varactor whose capacitance values can change along with the change of the reverse bias voltage. The designed VCO is simulated with the Agilent's advanced design system (ADS) software. The transient simulation in time domain and the harmonic balance simulation in frequency domain are run respectively to observe the signal waveform, the frequency spectrum, the output power and the phase noise of the oscillation signal. Finally, the VCO is measured on a spectrum analyzer and the test results show that the designed VCO has good characteristics during the frequency range of the LTE Band 40.

\section{Analysis Method and Circuit Structure}

The VCO is designed with the negative resistance analysis method [5 7] possessing the advantage of rapid start-up. Figure 1(a) shows the simplified model of a classic single port oscillator. 
On the basis of the theory of the negative resistance oscillator, the oscillation will occur when the total impedance of the circuit equals to zero, that is:

$$
Z_{T}(I, j \omega)=Z_{S}(I, j \omega)+Z_{L}(j \omega)=0
$$

where $Z_{T}(I, j \omega)$ is the total impedance of the circuit, $Z_{S}(I, j \omega)=R_{S}(I, j \omega)+j X_{S}(I, j \omega)$ is the input impedance of the active device and its real part and imaginary part are related to both the current and the frequency of the circuit, $Z_{L}(j \omega)=R_{L}+j X_{L}(j \omega)$ is the impedance of the passive load and its real part keeps constant and its imaginary part is only associated with the current of the circuit. Separating the real parts and the imaginary parts in Equation (1), the following equivalent equations can be achieved:

$$
\begin{aligned}
& R_{S}(I, j \omega)+R_{L}=0 \\
& X_{S}(I, j \omega)+X_{L}(j \omega)=0
\end{aligned}
$$

In an actual circuit, the real part of the impedance of the passive load is always positive, meaning that the active device should present an appropriate negative resistance according to the Equation (2) which mainly determines whether an oscillation can build up in the circuit. The positive resistance will consume power while the negative resistance can work as a signal source to provide power to the external circuit. When the oscillation occurs, the total resistance value needs to be less than 0 . Under this condition, there will be a continuous growing current corresponding to the frequency determined by Equation (3) in the circuit. As the current increases, the absolute value of the total resistance will decrease gradually until the current of the circuit has been a stable value, meaning that the total resistance value has reduced to zero.
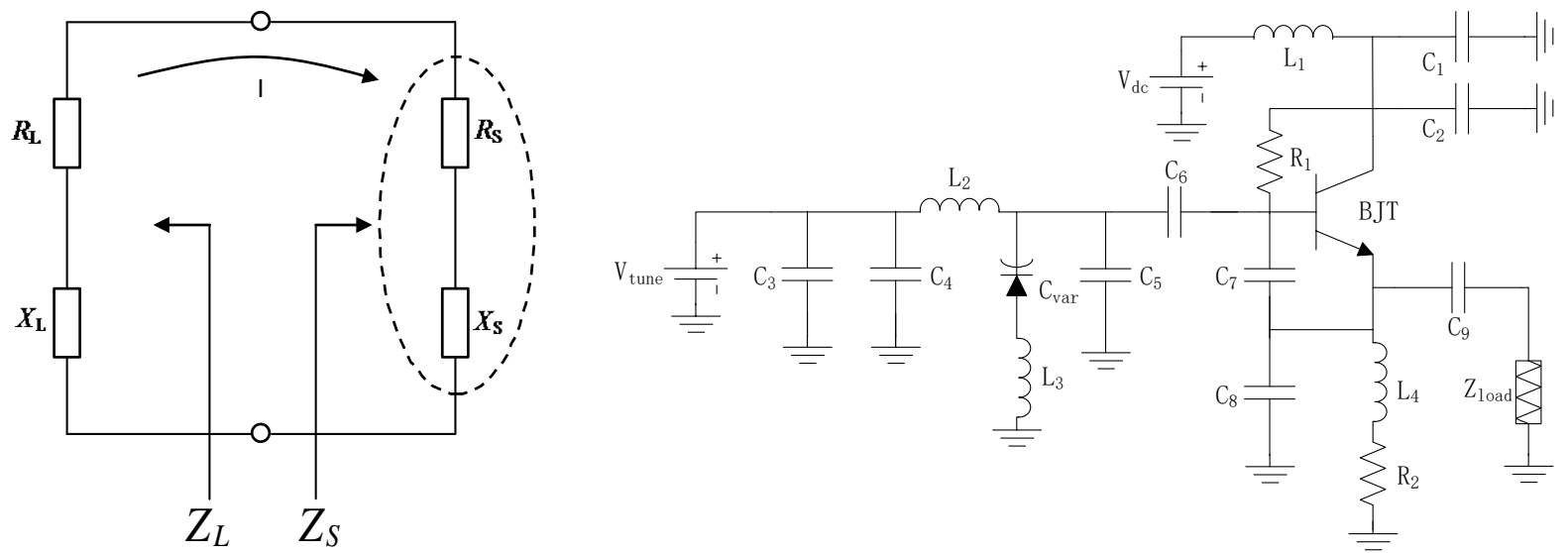

Fig.1. (a) The simplified model of the single port oscillator (b) The frame diagram of the VCO

The structure of the designed VCO in this paper is based on the modified Clapp oscillator and the circuit schematic is shown as Figure $1(b)$. In the circuit, $C_{1}, C_{2}, C_{3}$ and $C_{4}$ serve as $R F$ bypass capacitors to filter out the undesired noises introduced by the noisy DC power supply and the DC reverse bias voltage. Besides, $\mathrm{C}_{3}$ and $\mathrm{C}_{4}$ can provide an $\mathrm{RF}$ ground for the circuit at the collector of the BJT. The BJT is used in a common collector configuration and utilizes the feedback voltage divider $C_{7}-C_{8}$ to provide proper phase shift and voltage feedback to enable the oscillation to occur. In this case, the impedance seen from the base electrode of the BJT will be a negative value, meeting the start-up condition described by the negative-resistance analysis method. $L_{1}$ and $L_{2}$ are used as the RF chokes to isolate the RF signal, reducing the bad influences on the DC power supply introduced by the oscillation signal. $\mathrm{L}_{4}$ is also selected as the RF choke to provide a high impedance in the emitter circuit for the oscillation signal, ensuring that most power can be fed back to the base electrode of the BJT instead of being dissipated in $R_{2} . R_{2}$ is used as a current feedback device to provide the BJT a more stable static operating point. $\mathrm{C}_{7}, \mathrm{C}_{8}, \mathrm{C}_{\mathrm{var}}, \mathrm{L}_{3}$ and the junction capacitors in the BJT compose together the resonant circuit of the VCO, determining the oscillation frequency of the VCO. Tuning the reverse bias voltage $\mathrm{V}_{\text {tune }}$, the capacitance value of the varactor $\mathrm{C}_{\mathrm{var}}$ can be changed, resulting in corresponding change of the oscillation frequency. $\mathrm{C}_{6}$ is a coupling capacitor between the frequency tuning network and base electrode of the BJT. According to the Lesson's equation [8] which can be used to evaluate the phase noise performance of an oscillator, the circuit's 
loaded quality factor largely depends on $C_{5}$ and $C_{6}$ and has a great effect on the phase noise of the VCO. However, with the increased loaded quality factor, the power gain of the VCO circuit will be decreased, once the value of the gain is too small to compensate all kinds of losses caused by impedance mismatches, resistors and limited quality factor of the inductors and capacitors in the circuit, the VCO will not oscillate any more. Therefore, in order to meet the oscillation conditions and meanwhile to maintain good phase noise performance, there is often a trade-off between the loaded quality factor and the power gain of the VCO. $\mathrm{C}_{9}$ is a coupling capacitor which taps some of the energy out of the VCO to the load, and its value is mainly determined under the consideration of good impedance matching between the output impedance of the VCO and the load to achieve a high efficiency power transfer.

\section{Simulation Results}

Based on the negative resistance analysis method, the designed VCO as shown in Figure 1(b) is simulated in the ADS software. In order to evaluate the performance of the VCO, the transient simulation in time domain and the harmonic balance simulation in frequency domain are run to observe the frequency spectrum, the output power and the phase noise level of the oscillation signal. The values of the components in the circuit should be slightly adjusted according to their influences on the performance of different design indexes and the simulation results. As can be seen in Figure 2(a), the VCO can start up quickly and a stable oscillation can be reached in a short time.

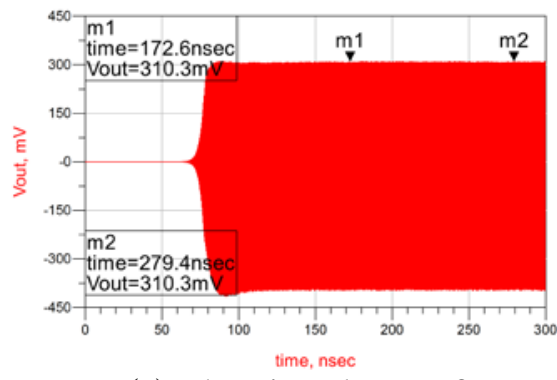

(a) The signal waveform

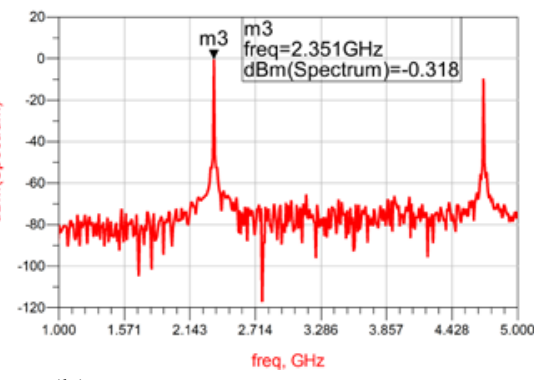

(b) The frequency spectrum

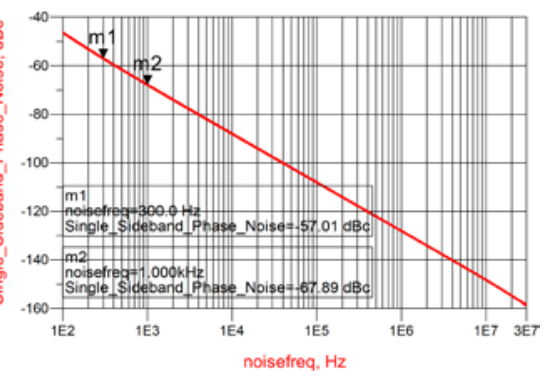

(c) The phase noise

Fig.2. Simulation results of the designed VCO

The simulated frequency spectrum of the designed VCO, as shown in Figure 2(b), indicates that the frequency of the fundamental oscillation is $2.351 \mathrm{GHz}$, closing to the mid-frequency of the LTE Band40, and the output power is $-0.318 \mathrm{dBm}$. In addition, the harmonic balance simulation is run to observe the phase noise performance and its simulation results are shown in Figure 2(c). The phase noises of the designed VCO are $-57.01 \mathrm{dBc} / \mathrm{Hz} @ 300 \mathrm{~Hz}$ and $-67.89 \mathrm{Bc} / \mathrm{Hz} @ 1 \mathrm{KHz}$, well meeting the low phase noise specification of the local RF oscillator in the systems of the mobile communication devices. During the process of simulations, the used varactor in the VCO circuit should be replaced by its equivalent circuit model which is normally a series RLC circuit [10], to improve the accuracy of the simulation results effectively. To evaluate the frequency tuning range of the designed VCO, the values of the capacitor in the varactor's model can be set to be a variable whose values are mainly determined by the range of the reverse bias voltage applied to the varactor.

\section{Measurement of the Designed VCO}

In order to meet the requirement of small size specification, all the passive components used in the VCO are chosen as 0402 SMT technology components and the circuit of the VCO is laid out in a tightly enclosed topology covered with a grounded metal shield. These behaviors result in a VCO with the size of $11 \mathrm{~mm}$ by $9 \mathrm{~mm}$, that is, $0.99 \mathrm{~cm}^{2}$. The photograph of the designed VCO is shown in Figure 4, compared by one RMB. The supply voltage of the VCO is generated by a high-efficiency and low noise voltage regulator. Due to the VCO operates at S band and the simulation is based on the ideal conditions without considerations of the parasitic effects, device tolerances, noisy DC power supply and other interference factors, the test results of the VCO using the same values of components achieved in the simulation could hardly be consistent with the simulation results. Thus 
the values of some components may be adjusted appropriately according to the test results.

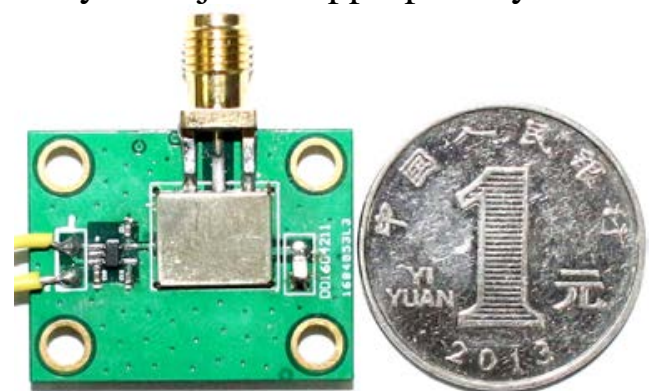

Fig.4. The photograph of the designed VCO compared by one RMB

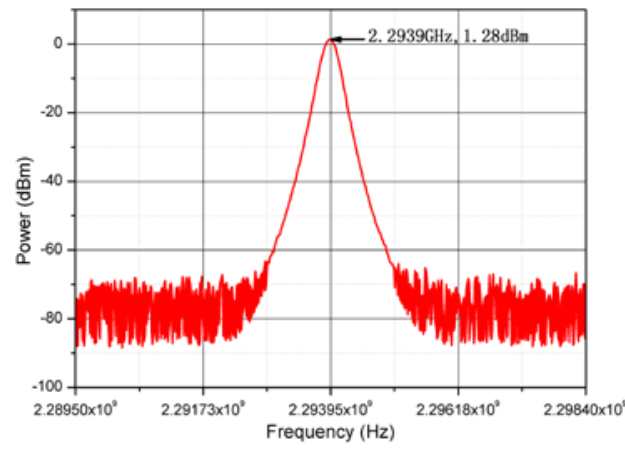

(a) The frequency spectrum

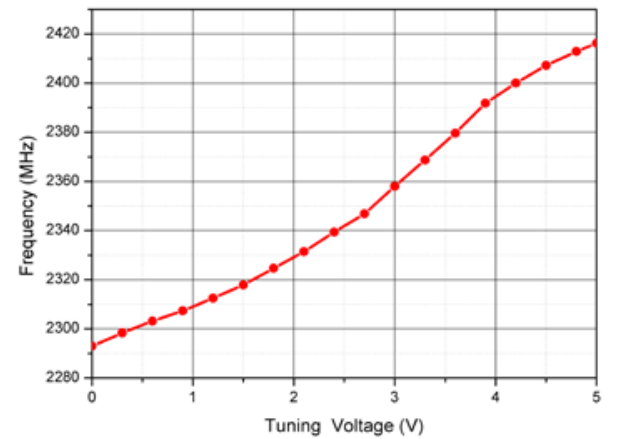

(c) The voltage-controlled tuning curve

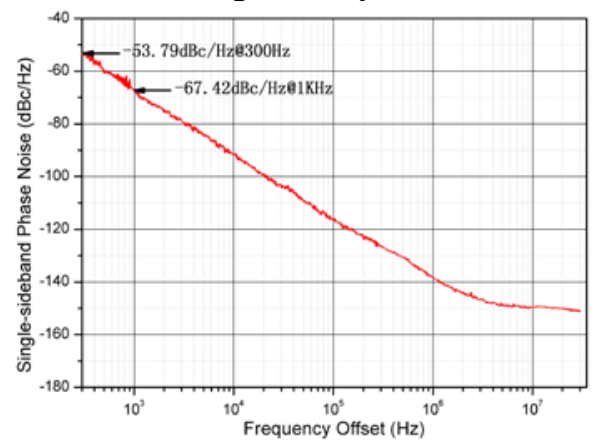

(b) The phase noise

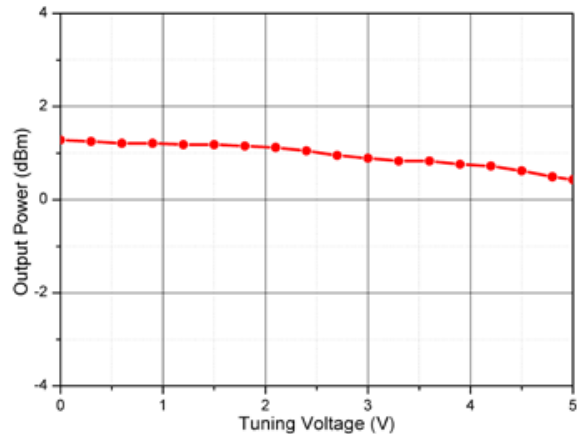

(d) The output power

Fig.5. The test results of the designed VCO

A spectrum analyzer is used to measure the performance of the designed VCO and the test results are shown in Figure 5(a) 5(d). Figure 5(a) shows that the frequency of the fundamental oscillation is about $2.2939 \mathrm{GHz}$ under the condition that the reverse bias voltage of the used varactor is equal to 0V. Figure 5(b) shows that the phase noises are $-53.79 \mathrm{dBc} / \mathrm{Hz} @ 300 \mathrm{~Hz}$ and $-67.42 \mathrm{dBc} / \mathrm{Hz} @ 1 \mathrm{KHz}$, meaning that the designed VCO has a good characteristic of low phase noise. Figure 5(c) shows that the voltage-controlled tuning sensitivity is about $24.4 \mathrm{MHz} / \mathrm{V}$ which results in a frequency tuning range from $2294 \mathrm{MHz}$ to $2416 \mathrm{MHz}$ with the reverse bias voltage of the varactor tuned from $0 \mathrm{~V}$ to $5 \mathrm{~V}$, covering the frequency range of the LTE Band40. Figure 5(d) shows that the output powers of the VCO with the varactor tuned at different reverse bias voltages. The total power consumption of the VCO is about $24 \mathrm{~mW}$.

\section{Conclusion}

A small size, low power consumption and low phase noise VCO used for the LTE Band40 is designed on the basis of the modified Clapp circuit topology and analyzed with the classic negative resistance analysis method. According to the 4G mobile communication frequency range distributed by Ministry of Industry and Information Technology of China, this VCO can be applied to the mixer system in the mobile phones which support the 4G LTE mode. With the ADS software, the transient simulation and the harmonic balance simulation are run to evaluate the oscillation frequency and the phase noise level of the VCO. Besides, the VCO is tested and the test results show that the VCO has an excellent performance, meeting the specifications of the $4 \mathrm{G}$ mobile communication well. 


\section{Acknowledgement}

In this paper, the research was sponsored by the National Natural Science Foundation of China (Grant No.11104313) and Sinoprobe-09-02-03.

The authors express thanks to Dr. Zhongshan Zhang from School of Electronic and Information Engineering, Soochow University, for his valuable suggestions about the test of the VCO.

\section{References}

[1] Willassen S Y. Forensics and the GSM Mobile Telephone System[J]. International Journal of Digital Evidence, 2003, 2(1):1-17.

[2] Salkintzis A. Interworking techniques and architectures for WLAN/3G integration toward 3G mobile data networks[J]. IEEE Wireless Communications, 2004, 11(3):50-61.

[3] Lin B S P, Tsai W H, Wu C C, et al. The Design of Cloud-Based 4G/LTE for Mobile Augmented Reality with Smart Mobile Devices[C]. 2013 IEEE Seventh International Symposium on Service-Oriented System Engineering. IEEE Computer Society, 2013:561-566.

[4] Sim C Y D, Lee Y L. Dual-band planar antenna for LTE 700/2300/2500 operation in laptop computer[C]. Electromagnetic Compatibility. IEEE, 2015.

[5] Kurokawa K. Some Basic Characteristics of Broadband Negative Resistance Oscillator Circuits[J]. Bell Labs Technical Journal, 1969, 48(6):1937-1955.

[6] Gonzalez G, Sosa O J. On the design of a series-feedback network in a transistor negative-resistance oscillator[J]. IEEE Transactions on Microwave Theory \& Techniques, 1999, 47(1):42-47.

[7] Everard J, Xu M, Bale, S. Simplified phase noise model for negative-resistance oscillators and a comparison with feedback oscillator models[J]. IEEE Transactions on Ultrasonics Ferroelectrics \& Frequency Control, 2012, 59(3):1-5.

[8] Leeson D B. A simple model of feedback oscillator noise spectrum[J]. Proceedings of the IEEE, 1966, 54(2):329-330.

[9] Jankovic M, Brannon A, Breitbarth J, et al. Design method for low-power, low phase noise voltage-controlled oscillators[C]. Microwave Integrated Circuit Conference, 2007. EuMIC 2007. European. 2007:199-202.

[10] Stauffer G H. Finding the Lumped Element Varactor Diode Model[J]. High Frequency Electronics, 2003:22-28. 